\title{
A practical nomogram for predicting cancer-specific survival in patients with clear-cell renal cell carcinoma
}

\section{Qian Wen}

The Ninth Hospital of Xi'an Affiliated Hospital of Xi'an Jiaotong University

\section{Xinwen Wang}

Xi'an Jiaotong University

\section{Xiaoye Wang}

The Ninth Hospital of Xi'an Affiliated Hospital of Xi'an Jiaotong University, Xi'an, Shaanxi, China;

\section{Tiao Bai}

4.The Ninth Hospital of Xi'an Affiliated Hospital of Xi'an Jiaotong University, Xi'an, Shaanxi, China;

Mei Tao ( $\nabla 49536573 @ q q . c o m$ )

The Ninth Hospital of Xi'an Affiliated Hospital of Xi'an Jiaotong University https://orcid.org/00000003-3431-211X

\section{Research article}

Keywords: Clear-cell renal cell carcinoma(ccRCC), Survival, Nomogram, SEER

Posted Date: August 12th, 2020

DOI: https://doi.org/10.21203/rs.2.24343/v2

License: (c) (i) This work is licensed under a Creative Commons Attribution 4.0 International License. Read Full License 


\section{Abstract}

Background: It has limitations in predicting patient cancer-specific survival to use of the traditional American Joint Committee on Cancer (AJCC) staging system alone.

Objectives: We aimed to establish and evaluate a comprehensive prognostic nomogram and compare its prognostic value with the AJCC-7 $7^{\text {th }}$ staging system in adults diagnosed with ccRCC.

Methods: We used the SEER database to identify 24477 cases of ccRCC between 2010 and 2015. In the development cohort, we used multivariate Cox proportional-hazards analyses to select significant variables, and used $\mathrm{R}$ software to establish a nomogram for predicting the 3-year and 5-year cancerspecific survival rates of cCRCC patients. In the development and validation cohorts, we compared our cancer-specific survival model with the AJCC- ${ }^{\text {th }}$ prognosis model to evaluate the performance of the nomogram by calculating the concordance index (C-index), Youden Index, area under the receiver operating characteristic curve (AUC), net reclassification improvement (NRI), and integrated discrimination improvement (IDI), and performing calibration plotting and decision curve analyses (DCAs).

Results: Eleven identified independent prognostic factors were used to establish the nomogram. Age at diagnosis, being unmarried, higher grades, larger tumor size, higher AJCC-7 ${ }^{\text {th }}$ stage, lymph node metastases, bone metastases, liver metastases, lung metastases, radiotherapy, and no surgery were risk factors for the cancer-specific survival of ccRCC. The C-index, Youden Index, AUC, NRI, IDI, and calibration plots demonstrated the good performance of the nomogram compared to the AJCC-7 ${ }^{\text {th }}$ staging system. Moreover, the 3-year and 5-year DCA curves showed that the nomogram yielded net benefits that were greater than the traditional AJCC-7 $7^{\text {th }}$ staging system.

Conclusion: This study is the first to indicate that married status is an important prognostic parameter in ccRCC. Our results also demonstrate that the developed nomogram can predict cancer-specific survival more accurately than the AJCC- $7^{\text {th }}$ staging system alone. The prognostic factors were easily obtained.

\section{Background}

Renal carcinoma accounts for around $3 \%$ of all adult malignancies ${ }^{1}$, and represents the tenth most common cancer in females and the sixth most common in males ${ }^{2}$. It caused an estimated 175,098 deaths $\left(1.8 \%\right.$ of the total cancer deaths) ever year ${ }^{3}$. Most (80-85\%) renal carcinomas are renal cell carcinoma (RCC), and they constitute the third most commonly diagnosed urogenital malignancy ${ }^{4}$. Clearcell renal cell carcinoma (ccRCC) patients constitute $80-90 \%$ of all RCC patients ${ }^{5}$. ccRCC is a potentially aggressive neoplasm reported to have an overall 5-year progression-free cancer-specific survival rate of $70 \%$ and a cancer-specific mortality rate of $24 \%{ }^{6}$. Establishing an effective prediction model can help clinicians to make clinical decisions. 
The American Joint Committee on Cancer (AJCC) staging system ${ }^{7}$ is a classification system for describing the extent of disease progression in cancer patients. It is based on the TNM stage that is generally believed the most powerful prognostic indicator for RCC, and it remains the most-used tool to classify RCC patients in clinical practice. However, research has shown that multivariate Cox proportionalhazards regression analyses including pathological and multiple clinical covariates were more accurate than the TNM stage in predicting patient cancer-specific survival ${ }^{8}$. Several pathology-based systems for predicting clinical outcomes, including those measuring gene expression, have been established to predict the prognosis of patients with RCC, such as the UISS (University of California Los Angeles integrated staging system), Mayo Clinic SSIGN (stage, size, grade, and necrosis) score, TNM stage, and TCGA (The Cancer Genome Atlas) ${ }^{9-12}$. However, these prediction models are based on difficult-to-obtain genetic data, have a low prediction accuracy, or lack systematic evaluations of the models on which they are based. Moreover, these are used to predict prognosis of patients with RCC rather than CcRCC.

We therefore aimed to establish a comprehensive prognostic nomogram and assumed it has better performance than the AJCC- $7^{\text {th }}$ classification in patients diagnosed with CCRCC.

\section{Methods}

\section{Patients}

Information about all of the included patients was retrieved from the latest version of the Surveillance, Epidemiology, and End Results (SEER) database. This study was approved by the Ethics Committee of the Ninth Hospital of Xi'an. The inclusion criteria were as follows:

- Renal carcinoma patients with an ICD-0-3/WHO 2008 histological type code of 8312/3 (ccRCC).

- Positive diagnostic confirmation in histology.

- Categorized as either alive or with thyroid carcinoma as the cause of death.

- Age at diagnosis of between 19 and 85 years.

The exclusion criteria were as follows:

- Unknown age, race, sex, marital status, insurance recode, tumor grade, tumor size, tumor site, AJCC$7^{\text {th }}$ stage, Mayo Clinic stage, surgery status, radiation status, chemotherapy status, lymph node metastases, bone metastases, brain metastases, liver metastases, or lung metastases, or incomplete SEER cause-specific death classification.

- Unknown survival time for a patient who was still alive.

- Diagnosis made by a death certificate or only an autopsy.

We collected the following data for each patient: age, race, sex, marital status, insurance recode, tumor grade, tumor size, tumor site, AJCC-7 ${ }^{\text {th }}$ stage, Mayo Clinic stage, surgery status, radiation status, chemotherapy status, lymph node metastases, bone metastases, brain metastases, liver metastases, lung 
metastases, and cancer-specific survival time (in months). The SEER cancer-specific death classification was the endpoint event. The application of the inclusion and exclusion criteria resulted in the identification of 24477 patients in the SEER database between 2010 and 2015, and the cases in this period were staged by AJCC 7th edition.

\section{Statistical analysis}

All variables are presented as median (25th-75th percentile) values because continuous variables such as age and cancer-specific survival time did not conform to a normal distribution. The Cox regression model analysis determined the hazard ratios (HRs) and 95\% confidence intervals (Cls).

Patients were randomly divided into a validation cohort (30\% of patients) and a development cohort ( $70 \%$ of patients) to use R software. In the development cohort, significant variables selected by Cox regression analysis $\llbracket$ stepwise $p<0.1 \rrbracket$ were used as predictors for the nomogram, which was established using $\mathrm{R}$ software. The nomogram was internally and externally validated in the development and validation cohorts, respectively.

To compare the discrimination performance of our nomogram with AJCC-7th modeling, we calculated Cindex, AUC which were widely used to evaluate the discrimination of prediction models ${ }^{13-15}$, and we also figured out sensitivity, specificity, positive predictive value, negative predictive value and Youden Index. We also evaluated the improvement in the predictive discrimination of our nomogram by calculating the relative integrated discrimination improvement (IDI) and the net reclassification improvement (NRI), as described by Pencina et al. ${ }^{16}$. Calibration plots were generated to evaluate the predictive accuracy by comparing the nomogram-predicted and actually observed 3-year and 5-year cancer-specific survival probabilities, as described by Vuk et al. and Cohen et al. ${ }^{17,18}$. We also estimated the clinical usefulness and net benefit of our nomogram using decision curve analysis (DCA), as described by Vickers et al. ${ }^{19}$.

All $P$ values were two-sided, with $P \leq 0.05$ considered statistically significant. The data were obtained using SEER* Stat version 8.3.5, and the statistical analyses were performed using SPSS version 21.0 and $\mathrm{R}$ software. Both discrimination and calibration were evaluated using bootstrapping with 500 resamples.

\section{Results}

\section{Clinicopathological characteristics}

The 24477 patients were divided into 17133 in the development cohort and 7344 in the validation cohort. The median age was 60 years in both cohorts. Most of the patients in the development and validation cohorts were white $(85.6 \%$ and $85.6 \%$, respectively), male $(62.2 \%$ and $61.2 \%)$, and married $(66.0 \%$ and $66.3 \%$ ). Most of the patients had insurance, a tumor size of $\leq 70 \mathrm{~mm}$, a tumor of grade II, AJCC-7 $7^{\text {th }}$ stage I, and localized Mayo Clinic stage, a tumor that had not metastasized to the lymph nodes, bone, brain, liver, or lung, and had received surgery but not radiation or chemotherapy. The cancer-specific survival 
time was 27 months in the development cohort and 28 months in the validation cohort, respectively. The demographics and tumor characteristics of patients are summarized in Table 1.

Table 1 Patients characteristics in the development cohort and validation cohorts. 
Development Cohort(N=17133) Validation Cohort

$(\mathrm{N}=7344)$

ian age at diagnosis, year (interquartile60(51-68)

$60(51-68)$

г)

n (\%)

le

$10651(62.2)$

$4493(61.2)$

nale

$6482(37.8)$

$2851(38.8)$

$\geqslant \mathrm{n}(\%)$

ite

$14666(85.6)$

1163(6.8)

$6286(85.6)$

ck

$1304(7.6)$

$512(7.0)$

ter $^{\mathrm{a}}$

$1304(7.6)$

$546(7.4)$

ital status n (\%)

rried

11301 (66.0)

$4870(66.3)$

married ${ }^{b}$

5832(34.0)

2474 (33.7)

rance Recode n (\%)

insurance

urance

584(3.4)

14481 (84.5)

$191(2.6)$

$\checkmark$ Medical

2068 (12.1)

6292(85.7)

le

Well differentiated)

(Moderately differentiated)

1797 (10.5)

9133 (53.3)

(Poorly differentiated)

4979(29.1)

(Undifferentiated; anaplastic)

$1224(7.1)$

861 (11.7)

or Size n (\%)

Jmm

13033(76.1)

$\mathrm{nm}-100 \mathrm{~mm}$

2555 (14.9)

j0mm

$1545(9.0)$

$752(10.2)$

3930 (53.5)

$2138(29.1)$

$524(7.1)$

- origin of primary

$8420(49.1)$

t - origin of primary

$8713(50.9)$

$5611(76.4)$

$1071(14.6)$

$662(9.0)$

$>-7^{\text {th }} \mathrm{n}(\%)$

$11188(65.3)$

$4849(66.0)$

$1612(9.4)$

$659(9.0)$

$3150(18.4)$

1347 (18.3)

1183(6.9)

3632 (49.5)

3712(50.5)

e n (\%)

ılized

$12800(74.7)$

3267(19.1)

$1066(6.2)$

489 (6.7)

onal

5508 (75.0)

1384(18.8)

$452(6.2)$

Modeling Cohort

Validation

Cohort

rery $\mathrm{n}(\%))$

17116(99.9)

7336

(99.9)

$17(0.1)$

$8(0.1)$

ation n (\%)

ne/Unknown

312 (1.8)

120 (1.6)

16821 (98.2)

7224

(98.4) 


\section{notherapy $\mathrm{n}(\%)$}

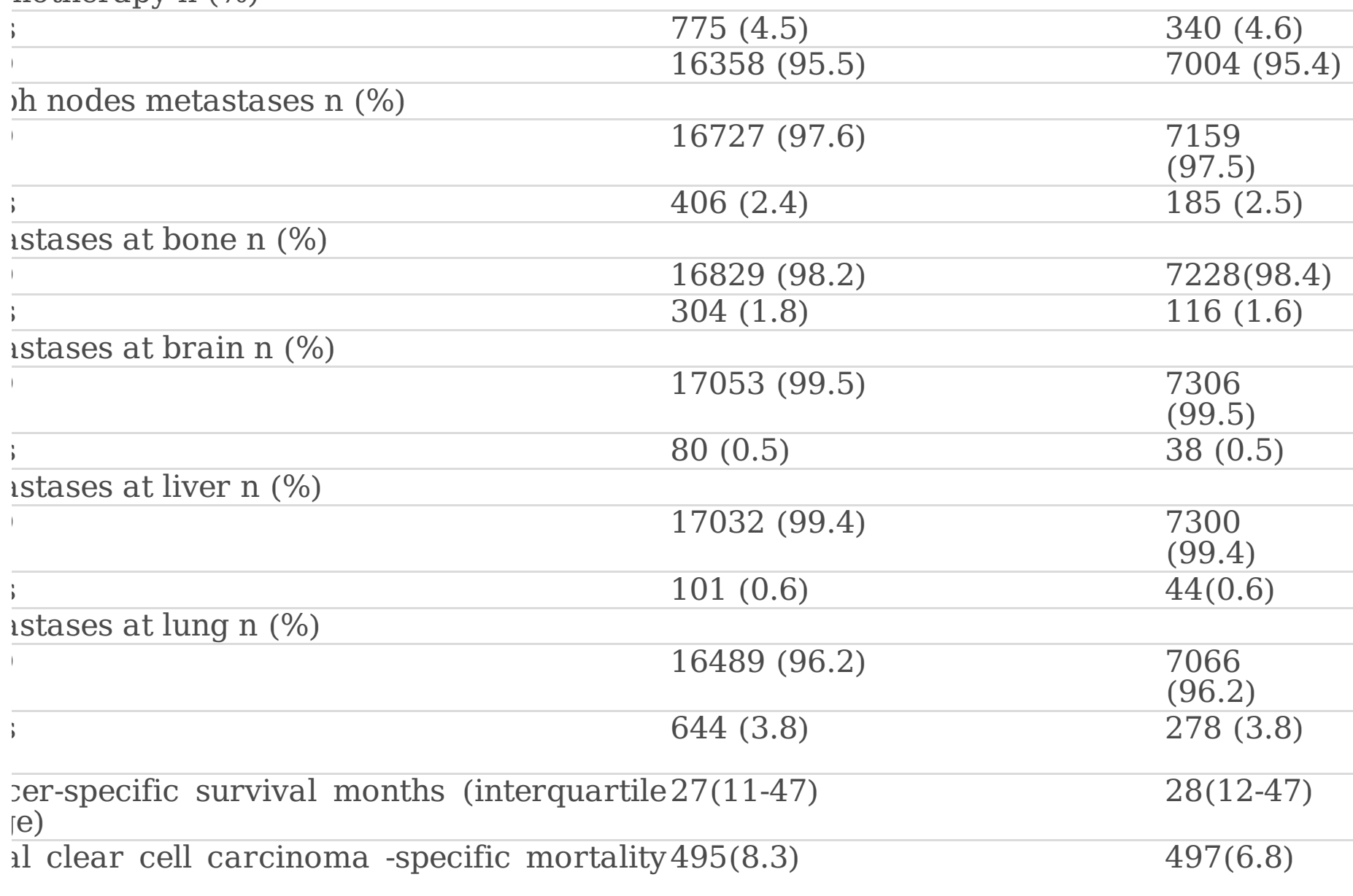

\section{a American Indian \& AK Native \& Asian \& Pacific Islander \\ b Single \& Separated \& Divorced \&Widowed \&Unmarried or Domestic Partner}

\section{Independent prognostic factors in the development cohort}

The variables of age at diagnosis, marital status, tumor grade, tumor size, AJCC-7 $7^{\text {th }}$ stage, surgery status, radiation status, lymph node metastases, bone metastases, liver metastases, and lung metastases were included in the multivariate Cox regression analyses in the development cohort. These multivariate analyses demonstrated that age at diagnosis ( $H R=1.0247, p<0.001)$, being unmarried $(H R=1.1515$ vs married, $p<0.01)$, grade II (HR=1.7572 vs grade I, $p<0.01)$, grade III (HR=3.3630 vs grade $\mathrm{I}, p<0.001)$, grade IV (HR=6.6275 vs grade $\mathrm{I}, p<0.001)$, tumor size $>50 \mathrm{~mm}$ and $<100 \mathrm{~mm}(\mathrm{HR}=1.4638$ vs tumor size $\leq 70 \mathrm{~mm}, p<0.001)$, tumor size $>100 \mathrm{~mm}$ (HR=1.8329 vs tumor size $\leq 70 \mathrm{~mm}, p<0.001)$, AJCC-7 ${ }^{\text {th }}$ stage II $\left(\mathrm{HR}=2.0843\right.$ vs AJCC- $7^{\text {th }}$ stage I, $\left.p<0.001\right)$, AJCC- $^{\text {th }}$ stage III (HR=4.3342 vs AJCC- $7^{\text {th }}$ stage $\left.\mathrm{l}, p<0.001\right)$, AJCC- $7^{\text {th }}$ stage IV (HR=10.2613 vs AJCC- $7^{\text {th }}$ stage $\left.I, p<0.001\right)$, no surgery (HR=4.9995 vs surgery, $p<0.001$ ), lymph node metastases ( $H R=1.7387 \mathrm{vs}$ no lymph node metastases, $p<0.001)$, bone metastases ( $H R=1.7746$ vs no bone metastases, $p<0.001)$, liver metastases $(H R=1.7064$ vs no liver metastases, $p<0.001)$, and lung metastases ( $\mathrm{HR}=1.6190$ vs no lung metastases, $p<0.001)$ were risk factors for cancer- 
specific survival. However, no radiation or any unknown radiation status $(\mathrm{HR}=0.6534$ vs radiation, $p<0.001$ ) was a protective factor for cancer-specific survival (Table 2).

Table 2 variables by multivariate Cox regression analysis (Development Cohort)

\begin{tabular}{|c|c|c|c|}
\hline \multirow[t]{2}{*}{ Variables } & \multicolumn{3}{|c|}{ Multivariate analysis } \\
\hline & HR & $95 \% \mathrm{CI}$ & P-value \\
\hline Age at diagnosis & 1.0247 & $1.0189-1.0306$ & $\square 0.001$ \\
\hline \multicolumn{4}{|l|}{ Marital status n (\%) } \\
\hline \multicolumn{4}{|c|}{ Reference } \\
\hline Unmarried & 1.1515 & $1.0170-1.3039$ & $\square 0.01$ \\
\hline \multicolumn{4}{|l|}{ Grade } \\
\hline I (Well differentiated) & & Reference & \\
\hline II (Moderately differentiated) & 1.7572 & $1.1076-2.7878$ & $\square 0.01$ \\
\hline III (Poorly differentiated) & 3.3630 & 2.1273-5.3166 & $\square 0.001$ \\
\hline IV (Undifferentiated; anaplastic) & 6.6275 & $4.1547-10.5721$ & $\square 0.001$ \\
\hline \multicolumn{4}{|l|}{ Tumor Size n (\%) } \\
\hline$\leq 70 \mathrm{~mm}$ & & Reference & \\
\hline 70mm-100mm & 1.4638 & $1.2231-1.7519$ & $\square 0.001$ \\
\hline$>100 \mathrm{~mm}$ & 1.8329 & 1.5334-2.1909 & $\square 0.001$ \\
\hline \multicolumn{4}{|l|}{ AJCC- $7^{\text {th }} \mathrm{n}(\%)$} \\
\hline I & & Reference & \\
\hline II & 2.0843 & $1.5187-2.8603$ & $\square 0.001$ \\
\hline III & 4.3342 & 3.4501-5.4449 & $\square 0.001$ \\
\hline IV & 10.2613 & 7.7868-13.5222 & $\square 0.001$ \\
\hline \multicolumn{4}{|l|}{ Surgery n (\%) } \\
\hline Yes & & Reference & \\
\hline NO & 4.9995 & $2.0409-12.2474$ & $\square 0.001$ \\
\hline \multicolumn{4}{|l|}{ Radiation n (\%) } \\
\hline Yes & & Reference & \\
\hline None/Unknown & 0.6534 & $0.5304-0.8048$ & $\square 0.001$ \\
\hline \multicolumn{4}{|l|}{ lymph nodes metastases n (\%) } \\
\hline NO & & Reference & \\
\hline Yes & 1.7387 & $1.4692-2.0576$ & $\square 0.001$ \\
\hline \multicolumn{4}{|l|}{ metastases at bone n (\%) } \\
\hline $\mathrm{NO}$ & & Reference & \\
\hline Yes & 1.7746 & $1.4167-2.2230$ & $\square 0.001$ \\
\hline \multicolumn{4}{|l|}{ metastases at liver n (\%) } \\
\hline NO & & Reference & \\
\hline Yes & 1.7064 & $1.3076-2.2269$ & $\square 0.001$ \\
\hline \multicolumn{4}{|l|}{ metastases at lung $\mathrm{n}(\%)$} \\
\hline $\mathrm{NO}$ & & Reference & \\
\hline Yes & 1.6190 & $1.3515-1.9395$ & $\square 0.001$ \\
\hline
\end{tabular}

Prognostic nomogram for 3-year and 5-year cancer-specific survival probabilities 
Age at diagnosis, marital status, tumor grade, tumor size, AJCC- $7^{\text {th }}$ stage, surgery status, radiation status, lymph node metastases, bone metastases, liver metastases, and lung metastases were significant predictors for ccRCC in the development cohort (Table 2). These variables were used to develop the predictive nomogram (Fig. 1).

\section{Validation of the prognostic nomogram}

We used the C-index, Youden Index, AUC, NRI, and IDI to assess the discrimination performance of the nomogram. The C-index was higher for the nomogram than for the AJCC-7th staging system both in the development cohort ( 0.898 vs 0.856 ) and in the validation cohort ( 0.905 vs 0.862$)$. Youden Index was higher for the nomogram than for the AJCC-7th staging system both in the development cohort and in the validation cohort( Table 3 and 4). Both 3-year and 5-year cancer-specific survival outcomes that the sensitivity, specificity, positive predictive value, negative predictive value were compared between the nomogram and AJCC.( Table 3 and 4). The AUC was better for the nomogram than for the AJCC-7 ${ }^{\text {th }}$ model in both the development and validation cohorts (Figure 2). Comparing with the AJCC-7 ${ }^{\text {th }}$ staging system, the 3-year and 5-year NRI values for the nomogram were $0.276(95 \% \mathrm{Cl}=0.214-0.328)$ and 0.284 (95\% $\mathrm{Cl}=0.230-0.352)$, respectively, in the development cohort, and $0.263(95 \% \mathrm{Cl}=0.161-0.350)$ and 0.339 (95\% $\mathrm{Cl}=0.234-0.408)$ in the validation cohort. Comparing with the AJCC- $7^{\text {th }}$ staging system, the $3-$ year and 5-year IDI values for the nomogram were 0.060 and 0.052 , respectively, in the development cohort, and 0.046 and 0.054 in the validation cohort.

Calibration plots of the nomogram showed that the predicted 3-year and 5-year cancer-specific survival probabilities of the model were almost identical to the actual observations in both the development and validation cohorts (Figure 3). The 3-year and 5-year DCA curves for the nomogram demonstrated net benefits that were greater than those for the traditional AJCC- $7^{\text {th }}$ staging system in both the development set and the validation set, although both models demonstrated net benefits (Figure 4).

Table 3 Performance of the nomogram' probability for 3-year cancer-specific survival

\begin{tabular}{lcccc}
\hline & \multicolumn{2}{c}{ Modeling Cohort } & \multicolumn{2}{c}{ Validation Cohort } \\
& the nomogram & AJCC-7th & the nomogram & AJCC-7th \\
\hline sensitivity(\%) & 88.87 & 80.97 & 89.07 & 80.21 \\
specificity(\%) & 76.64 & 79.69 & 78.47 & 80.08 \\
\hline positive predictive value(\%) & 95.7 & 96.89 & 95.83 & 95.73 \\
\hline negative predictive value(\%) & 54.09 & 41.74 & 56.36 & 42.12 \\
\hline Youden Index(\%) & 65.51 & 60.66 & 67.54 & 60.29 \\
\hline
\end{tabular}

Table 4 Performance of the nomogram' probability for 5-year cancer-specific survival 


\begin{tabular}{lcccc}
\hline & \multicolumn{2}{c}{ Modeling Cohort } & \multicolumn{2}{c}{ Validation Cohort } \\
& the nomogram & AJCC-7th & the nomogram & AJCC-7th \\
\hline sensitivity(\%) & 88.61 & 81.42 & 89.23 & 83.13 \\
specificity(\%) & 78.17 & 79.69 & 79.88 & 80.08 \\
\hline positive predictive value(\%) & 87.57 & 87.44 & 88.18 & 87.53 \\
\hline negative predictive value(\%) & 79.82 & 71.19 & 81.52 & 73.84 \\
\hline Youden Index(\%) & 66.78 & 61.11 & 69.11 & 63.21 \\
\hline
\end{tabular}

\section{Discussion}

In addition to histological grade, the tumor size, Mayo Clinic stage at presentation, vascular invasion, and tumor necrosis are prognostic factors that are routinely utilized to predict the ultimate patient cancerspecific survival ${ }^{20,21}$. We found that age at diagnosis, being unmarried, and metastases in the lymph nodes, bone, liver, and lung were risk factors for cancer-specific survival. In particular, this is the first study to include a married status in a cancer-specific survival prediction model of ccRCC. The risk of death increased with Mayo Clinic stage, AJCC-7 ${ }^{\text {th }}$ stage, and tumor size.

As is well known, surgery remains the most important and probably the only curative approach in cCRCC $^{22}$. Our study found that surgery can improve the prognosis of $c C R C C$, whereas radiation therapy is a risk factor for cancer-specific survival. This might be due to radiotherapy long being considered a valueless approach for managing primary disease, and so mainly being prescribed to treat distant metastases, especially brain and painful bone metastases, with a palliative intent ${ }^{2324}$. Moreover, patients with radiotherapy were in a more advanced state or had metastases comparing with patients without radiotherapy. Therefore, the prognosis of radiotherapy patients was worse than that of patients who had not received radiotherapy.

Nomograms have been used in most cancer types in recent years ${ }^{25-32}$, including for ccRCC ${ }^{33-35}$. However, there has been a lack of overall evaluations of the developed nomograms, or the variables used for prediction have not been readily available. The clinical applicability and ease of use are highly attractive features of the comprehensive prognostic nomogram we constructed in this study, and we have compared its prognostic value with that of the AJCC- $7^{\text {th }}$ classification. Our nomogram model contains risk factors that are easy to obtain from historical records.

To further determine whether the prognostic model performed better than the traditional AJCC-7 $7^{\text {th }}$ staging system, we evaluated the performance of our cancer-specific survival model using several basic features of model validation: C-index, Youden Index, AUC, NRI, IDI, calibration plots, and DCA. The AUC ${ }^{36}$ or Cstatistic $^{37}$ is typically used to assess the discrimination performance. The IDI and categorical NRI were also used to assess discrimination in terms of the additional diagnostic value of our model compared to the AJCC-7 ${ }^{\text {th }}$ model. All of these indicators showed that our model has better discrimination performance than the AJCC- $7^{\text {th }}$ staging system. The calibration plots resembled a 45-degree line, indicating that the 
nomogram predictions were well calibrated (Figure 3). DCA is used to evaluate clinical usefulness, and it shows the minimal net benefit of modified scores that incorporate an index. Some studies have demonstrated the benefits of DCA and recommended its use ${ }^{38,39}$. The present results for the 3-year and 5year DCA curves showed that our model yielded net benefits that were greater than those for the traditional AJCC- $7^{\text {th }}$ staging system in both the development and validation cohorts (Figure 4).

The above-described findings indicate that using our new nomogram can ameliorate the gap that exists relative to predictions based on the AJCC- $7^{\text {th }}$ staging system alone. This supports that our nomogram is a useful tool for optimizing treatment in the clinical setting of ccRCC.

\section{Limitations}

This study was subject to some limitations. The patients were mainly white, and so the results might not be applicable to other racial groups. Our data set and follow-up data came from the SEER database, which is retrospective and so has inevitable inherent bias. There was also selection bias in the selection and exclusion of patients, because we only selected the patients with complete information. In addition, many factors were not included, such as the statuses of VEGF, HIF-1a, HIF-2a, p53, and Ki-6740,41, which have been shown to influence the prognosis of ccRCC. Because the included subjects had a higher survival rate and fewer subjects died during the follow-up period, the Validation curve only showed the portion with high survival rate. Another limitation of this study is the relatively small sample, and so more data are needed to provide more accurate performance assessments of the model. Finally, the predicted values calculated from the nomogram are for reference use by clinicians only, and the nomogram should be externally validated in another population in the future.

\section{Conclusions}

This study is the first to indicate that married status is an important prognostic parameter in ccRCC. Our results also demonstrate that the developed nomogram can predict cancer-specific survival more accurately than the AJCC-7 $7^{\text {th }}$ staging system alone. The prognostic factors were easily obtained. The nomogram could provide predictions for individual ccRCC patients and help clinicians in decision-making about treatment options and prognosis evaluations.

\section{Declarations}

\section{Acknowledgments}

We thank all colleagues involved in the study for their contributions.

\section{Funding}

None. 
Availability of data and material

The datasets analyzed during current study are available from the corresponding author upon reasonable request.

\section{Authors' contributions}

(1) Conception and design: Xinwen Wang and Qian Wen. (2) Administrative support: Tao Mei and Xiaoye Wang. (3) Provision of study materials or patients: Tiao Bai. (4) Collection and assembly of data: Qian Wen and Xinwen Wang. (5) Data analysis and interpretation: Qian Wen and Xinwen Wang. (6) Manuscript writing: Qian Wen and Xinwen Wang. (7) Final approval of manuscript: Qian Wen, Xinwen Wang, Xiaoye Wang, Tiao Bai and Tao Mei.

\section{Ethics approval and consent to participate}

This study was approved by the Ethics Committee of the Ninth Hospital of Xi'an

\section{Consent for publication}

All patients came from the SEER database (Surveillance, Epidemiology, and End Result), which is publicly available.

\section{Competing interests}

The author reports have no conflicts of interest in this work.

\section{References}

1. Remon J, Lianes P, Martinez S. Brain metastases from renal cell carcinoma. Should we change the current standard? CANCER TREAT REV. 2012 2012-06-01;38(4):249-57.

2. Siegel RL, Miller KD, Jemal A. Cancer statistics, 2018. CA Cancer J Clin. 2018 2018-01-01;68(1):7-30.

3. Bray F, Ferlay J, Soerjomataram I, Siegel RL, Torre LA, Jemal A. Global cancer statistics 2018 : GLOBOCAN estimates of incidence and mortality worldwide for 36 cancers in 185 countries. CA Cancer J Clin. 2018 2018-11-01;68(6):394-424.

4. Landis SH, Murray T, Bolden S, Wingo PA. Cancer statistics, 1999. CA Cancer J Clin. 1999 1999-0101;49(1):8-31, 1.

5. Ljungberg B, Bensalah K, Canfield S, et al. EAU guidelines on renal cell carcinoma: 2014 update. EUR UROL. 2015 2015-05-01;67(5):913-24.

6. Amin MB, Amin MB, Tamboli P, et al. Prognostic impact of histologic subtyping of adult renal epithelial neoplasms: an experience of 405 cases. AM J SURG PATHOL. 2002 2002-03-01;26(3):28191. 
7. Meng ZW, Pan W, Hong HJ, Chen JZ, Chen YL. Modified staging classification for intrahepatic cholangiocarcinoma based on the sixth and seventh editions of the AJCC/UICC TNM staging systems. Medicine (Baltimore). 2017 2017-08-01;96(34):e7891.

8. Moch H, Artibani W, Delahunt B, et al. Reassessing the Current UICC/AJCC TNM Staging for Renal Cell Carcinoma. EUR UROL. 2009;56(4):636-43.

9. Li P, Ren H, Zhang Y, Zhou Z. Fifteen-gene expression based model predicts the survival of clear cell renal cell carcinoma. MEDICINE. 2018;97(33):e11839.

10. Ficarra V, Novara G, Galfano A, et al. The 'Stage, Size, Grade and Necrosis' score is more accurate than the University of California Los Angeles Integrated Staging System for predicting cancerspecific survival in patients with clear cell renal cell carcinoma. BJU INT. 2009;103(2):165-70.

11. Leibovich BC, Blute ML, Cheville JC, et al. Prediction of progression after radical nephrectomy for patients with clear cell renal cell carcinoma: a stratification tool for prospective clinical trials. CANCER-AM CANCER SOC. 2003 2003-04-01;97(7):1663-71.

12. Frank I, Blute ML, Cheville JC, Lohse CM, Weaver AL, Zincke H. An outcome prediction model for patients with clear cell renal cell carcinoma treated with radical nephrectomy based on tumor stage, size, grade and necrosis: the SSIGN score. J Urol. 2002 2002-12-01;168(6):2395-400.

13. Dai W, Mo S, Xiang W, et al. The Critical Role of Tumor Size in Predicting Prognosis for T1 Colon Cancer. ONCOLOGIST. 2019 2019-11-20.

14. Bajaj S, Donnelly D, Call M, et al. Melanoma Prognosis - Accuracy of the American Joint Committee on Cancer Staging Manual Eighth Edition. J Natl Cancer Inst. 2020 2020-01-24.

15. Wu J, Zhang H, Li L, et al. A nomogram for predicting overall survival in patients with low-grade endometrial stromal sarcoma: A population-based analysis. Cancer Commun (Lond). 2020 2020-0618.

16. Pencina MJ, D'Agostino RB, Steyerberg EW. Extensions of net reclassification improvement calculations to measure usefulness of new biomarkers. STAT MED. 2011 2011-01-15;30(1):11-21.

17. Vuk M, Curk T. ROC Curve, Lift Chart and Calibration Plot. Metodoloski Zvezki. 2006 2006-01-01;3(1 ):89.

18. Cohen I, Goldszmidt M: Properties and Benefits of Calibrated Classifiers: Springer Berlin Heidelberg; 2004

19. Vickers AJ, Elkin EB. Decision Curve Analysis: A Novel Method for Evaluating Prediction Models. MED DECIS MAKING. 2016;26(6):565-74.

20. Delahunt B, Cheville JC, Martignoni G, et al. The International Society of Urological Pathology (ISUP) grading system for renal cell carcinoma and other prognostic parameters. AM J SURG PATHOL. 2013 2013-10-01;37(10):1490-504.

21. Delahunt B, Bethwaite PB, Nacey JN. Outcome prediction for renal cell carcinoma: evaluation of prognostic factors for tumours divided according to histological subtype. PATHOLOGY. 2007 200710-01;39(5):459-65. 
22. Bamias A, Escudier B, Sternberg CN, et al. Current Clinical Practice Guidelines for the Treatment of Renal Cell Carcinoma: A Systematic Review and Critical Evaluation. ONCOLOGIST. 2017 2017-0601;22(6):667-79.

23. Motzer RJ, Jonasch E, Agarwal N, et al. Kidney Cancer, Version 2.2017, NCCN Clinical Practice Guidelines in Oncology. J Natl Compr Canc Netw. 2017 2017-06-01;15(6):804-34.

24. De Felice F, Tombolini V. Radiation therapy in renal cell carcinoma. Critical Reviews in Oncology/Hematology. 2018;128:82-7.

25. Tan X, Ma Z, Yan L, Ye W, Liu Z, Liang C. Radiomics nomogram outperforms size criteria in discriminating lymph node metastasis in resectable esophageal squamous cell carcinoma. EUR RADIOL. 2018 2018-06-19.

26. Wang Y, Guan Q, Xiang J. Nomogram for predicting central lymph node metastasis in papillary thyroid microcarcinoma: A retrospective cohort study of 8668 patients. INT J SURG. 2018 2018-0701;55:98-102.

27. Chen $Y$, Zhang $Y$, Yang W, et al. Accuracy of a nomogram to predict the survival benefit of surgical axillary staging in T1 breast cancer patients. MEDICINE. 2018;97(26):e11273.

28. Ó Hartaigh BP, Gransar HM, Callister TM, et al. Development and Validation of a Simple-to-Use Nomogram for Predicting 5-, 10-, and 15-Year Survival in Asymptomatic Adults Undergoing Coronary Artery Calcium Scoring. JACC: Cardiovascular Imaging. 2017;11(3 ):450-8.

29. Ge MH, Cao J, Wang JY, et al. Nomograms predicting disease-specific regional recurrence and distant recurrence of papillary thyroid carcinoma following partial or total thyroidectomy. Medicine (Baltimore). 2017 2017-07-01;96(30):e7575.

30. Kim SK, Chai YJ, Park I, et al. Nomogram for predicting central node metastasis in papillary thyroid carcinoma. J SURG ONCOL. 2017 2017-03-01;115(3):266-72.

31. Wen J, Yang Y, Liu P, et al. Development and validation of a nomogram for predicting survival on the base of modified lymph node ratio in breast cancer patients. The Breast. 2017;33:14-22.

32. Zeng $\mathrm{Q}$, Hong $\mathrm{MH}$, Shen $\mathrm{LJ}$, et al. Nomograms for predicting long-term survival in patients with nonmetastatic nasopharyngeal carcinoma in an endemic area. Oncotarget. 2016 2016-0517;7(20):29708-19.

33. Chang Y, Xu L, Zhou L, et al. Granulocyte macrophage colony-stimulating factor predicts postoperative recurrence of clear-cell renal cell carcinoma. Oncotarget. 2016 2016-0426;7(17):24527-36.

34. Xu Z, Liu Y, Yang Y, et al. High expression of Mucin13 associates with grimmer postoperative prognosis of patients with non-metastatic clear-cell renal cell carcinoma. Oncotarget. 2017 2017-0131;8(5):7548-58.

35. Zhou L, Chang Y, Xu L, et al. The Presence of Vascular Mimicry Predicts High Risk of Clear Cell Renal Cell Carcinoma after Radical Nephrectomy. J Urol. 2016 2016-08-01;196(2):335-42.

36. The Meaning under a Receiver Characteristic and Use of the Area Operating (ROC) Curve1. 
37. Pencina MJ, D'Agostino RS. Evaluating Discrimination of Risk Prediction Models: The C Statistic. JAMA. 2015 2015-09-08;314(10):1063-4.

38. Talluri R, Shete $S$. Using the weighted area under the net benefit curve for decision curve analysis. BMC Med Inform Decis Mak. 2016 2016-07-18;16:94.

39. Rousson V, Zumbrunn T. Decision curve analysis revisited: overall net benefit, relationships to ROC curve analysis, and application to case-control studies. BMC Med Inform Decis Mak. 2011 2011-0622;11:45.

40. Ebru T, Fulya OP, Hakan A, et al. Analysis of various potential prognostic markers and survival data in clear cell renal cell carcinoma. INT BRAZ J UROL. 2017 2017-05-01;43(3):440-54.

41. Fan Y, Li H, Ma X, et al. Prognostic Significance of Hypoxia-Inducible Factor Expression in Renal Cell Carcinoma: A PRISMA-compliant Systematic Review and Meta-Analysis. Medicine (Baltimore). 2015 2015-09-01;94(38):e1646.

\section{Figures}

Points
Age
Mari
Grade
Size
AJCC
SUR
RAD
LYM
M.bo
M.Li
M.lu
Total Points
3-years Survival Probability
5-years Survival Probability

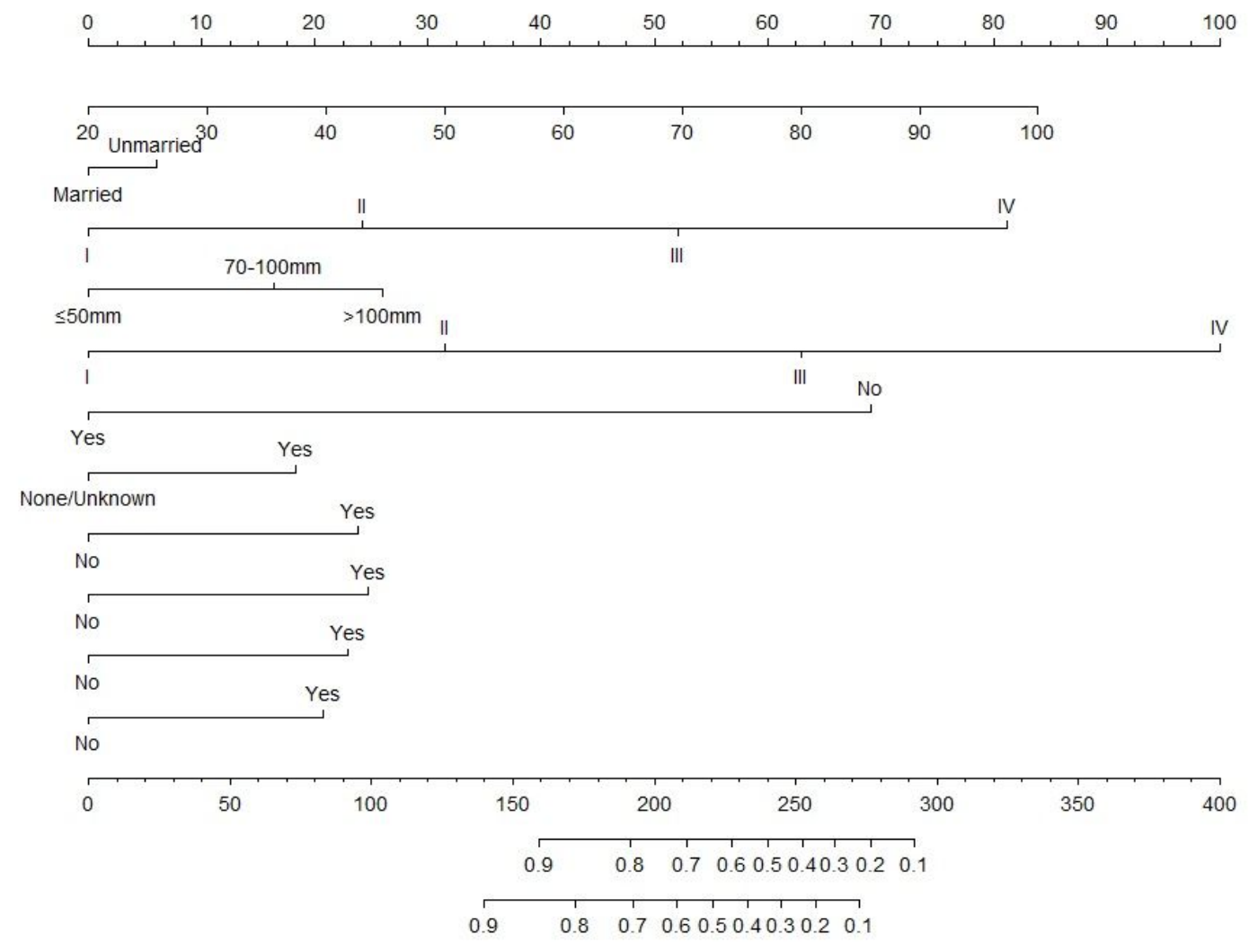

\section{Figure 1}


Nomogram predicting 3-year and 5-year cancer-specific survival. Mari: Marital status. Unmarried: Single \& Separated \& Divorced \&Widowed \&Unmarried or Domestic Partner. SUR: Surgery. RAD: Radiation. LYM: lymph nodes metastases.M.bo: metastases at bone. M.Ii: metastases at liver. M.lu: metastases at lung.
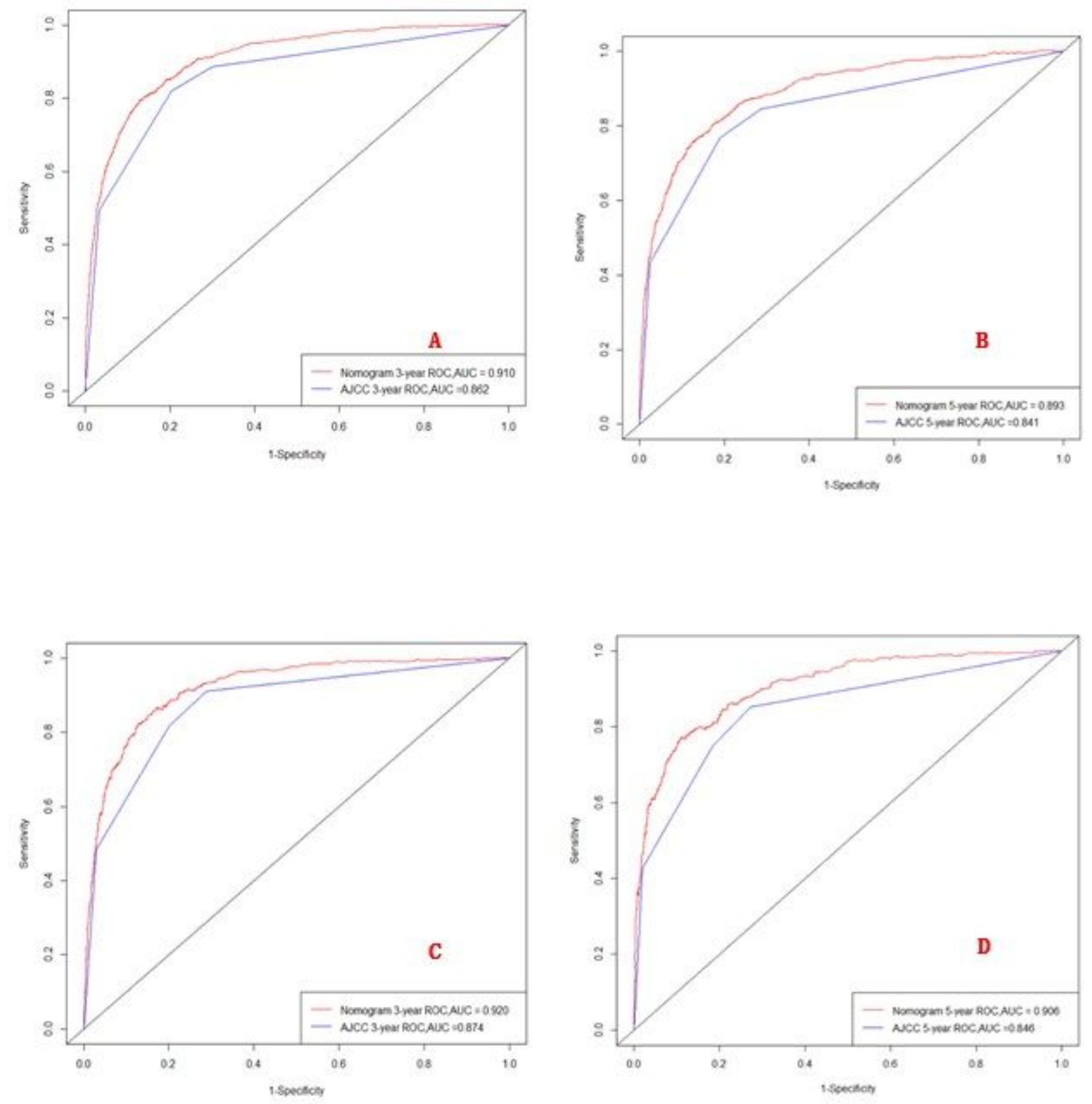

\section{Figure 2}

Area under the receiver operating characteristic curve (AUC). A, B came from the development set, and C, D came from the validation set. 

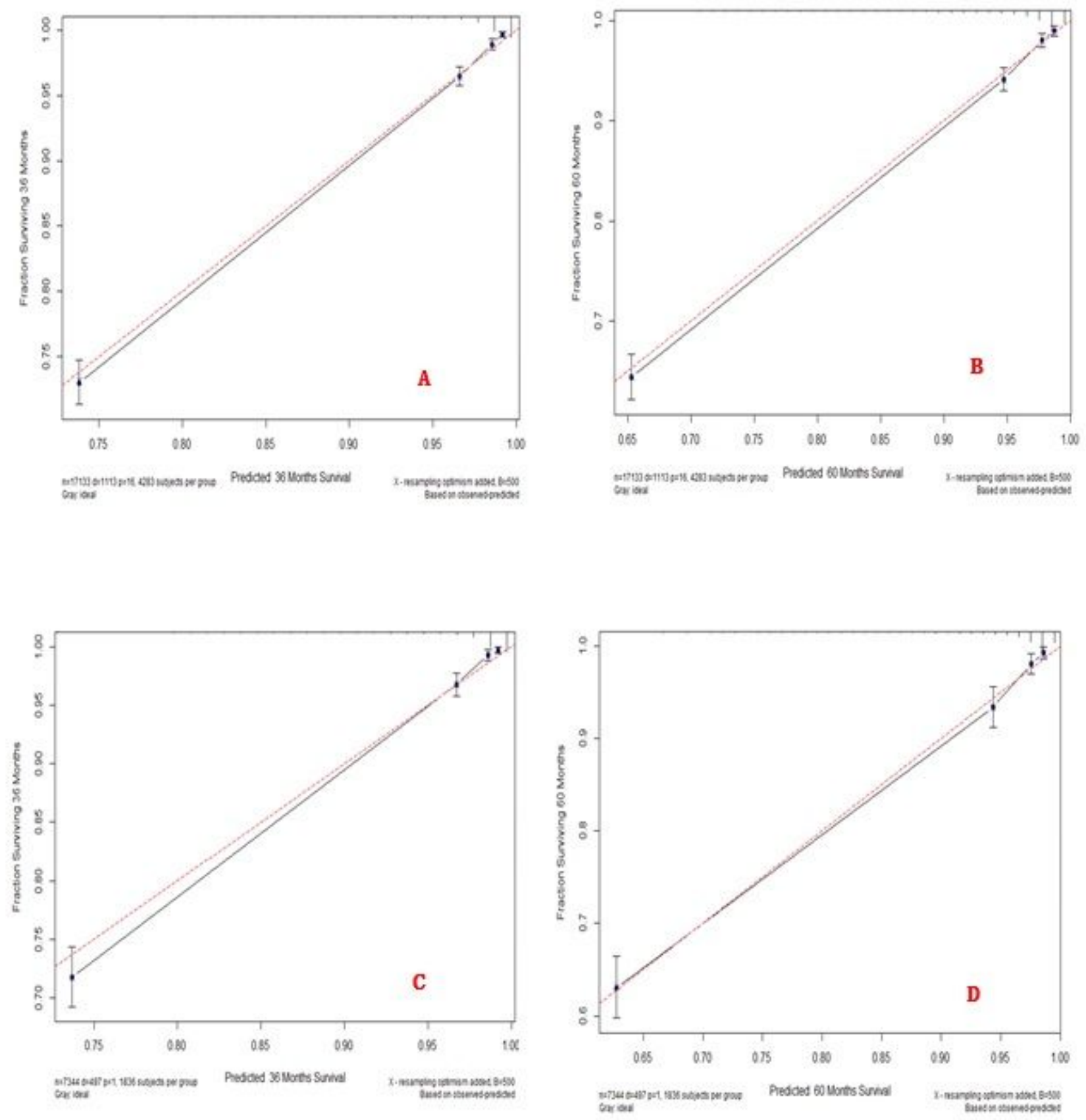

Figure 3

Calibration plots. Show the relationship between the predicted probabilities for 3-and 5-years cancerspecific survival base on the nomogram and actual values in the Validation sets. On the calibration curve, $\mathrm{x}$-axis is nomogram predicted probability of cancer-specific survival, and $\mathrm{y}$-axis is observed cancerspecific survival. ( $A, B$ in the development set and $C, D$ in the validation set ) 

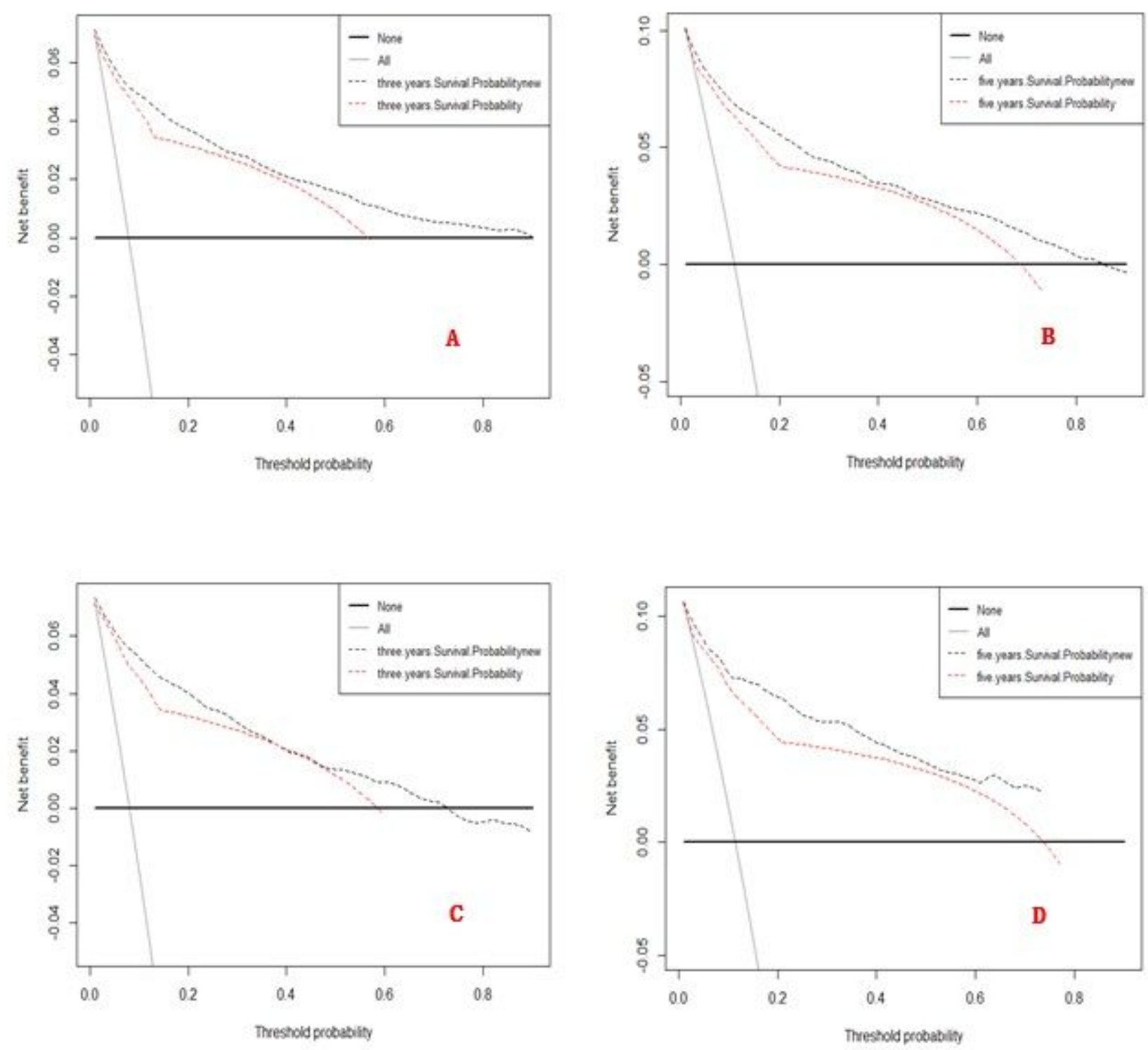

Figure 4

Decision curve analysis in the figure. The abscissa is the threshold probability, the ordinate is the net benefit rate. The horizontal one indicates that all samples are negative and all are not treated, with a net benefit of zero. The oblique one indicates that all samples are positive. The net benefit is a backslash with a negative slope. A, B show prediction for 3-and 5-years cancer-specific survival in the development sets. C, D show prediction for 3- and 5-years cancer-specific survival in the Validation sets. Cancerspecific survival probability new: the nomogram. Cancer-specific survival probability: AJCC-7th. 